\title{
El desempeño laboral en organizaciones públicas y privadas
}

\section{Labor performance in public and private organizations}

\author{
${\text { Marco Lluncor }{ }^{1} \text { (D)., Adolfo Cacho }}^{2}$ (D)., Walter Zegarra ${ }^{3}$ (D). y Delicia Olano ${ }^{4}$ (D).
}

\section{RESUMEN}

El objetivo de la investigación fue evaluar las competencias de los servidores como factor clave de su rendimiento productivo en diversas organizaciones. El estudio fue descriptivo de corte transversal con enfoque cuantitativo y diseño no experimental, donde la muestra ha sido no probabilística, se ha empleado la técnica de la encuesta y el instrumento un cuestionario con escala tipo Likert; los datos se analizaron a través del paquete estadístico SPSS v. 20 y 25 . Los resultados muestran que las características organizacionales y el desempeño laboral obtienen un valor general $\mathrm{p}=0,36$, la influencia de la jefatura y el desempeño laboral obtuvo en general un valor $\mathrm{p}=0,51$ y los motivadores del trabajo con el desempeño laboral, tuvo un valor general $\mathrm{p}=0,15$. Además, los servidores públicos o privados poseen un desempeño laboral suficiente, siendo necesario que la alta dirección de las organizaciones retomar la implementación de un programa de evaluación periódica de los servidores, programas de adiestramiento de capacidades que contribuyan a fortalecer sus conocimientos y prácticas del buen uso laboral.

Palabras clave: Desempeño laboral, evaluación, programa de adiestramiento.

\begin{abstract}
An exhaustive analysis of work performance in public and private organizations was carried out, the objective was to evaluate the competencies of the employees as a key factor of their productive performance in different organizations, the studies were characterized by being descriptive of transversal cut with quantitative approach and non-experimental design, the sample applied was non-probabilistic by convenience, applying the survey technique and the instrument was the questionnaire with Likert type scale, all the data was analyzed through the statistical package SPSS v. 20 and 25. 20 and 25. The results show that the organizational characteristics and work performance obtained a general value $\mathrm{p}=0.36$, the influence of the boss and work performance obtained in general a value $p=0.51$ and the motivators of the work with work performance, had a general value $\mathrm{p}=0.15$. The results show that public or private employees have a sufficient work performance, and it is necessary for the top management of the organizations to resume the implementation of a periodic evaluation program for employees, training programs that contribute to strengthen their knowledge and practices of good work use.
\end{abstract}

Keywords: job performance, evaluation, training program.

DOI: https://doi.org/10.37787/pakamuros-unj.v9i1.159

Recibido: 22/03/2020. Aceptado: 21/11/2020

* Autor para correspondencia

1. Doctorando Universidad Cesar Vallejo-Chiclayo, Perú. Email: marco.lluncor@gmail.com

2. Doctorando Universidad Cesar Vallejo-Chiclayo, Perú. Email: acachorevilla@gmail.com

3. Doctorando Universidad Cesar Vallejo-Chiclayo, Perú. Email: waltereloy.19@gmail.com

4. Doctorando Universidad Cesar Vallejo-Chiclayo, Perú. Email: delly_Ola@live.com 


\section{INTRODUCCIÓN}

Para desarrollar un buen desempeño laboral, existen otros factores como el liderazgo de los directivos, la colaboración, el trabajo en equipo, la carrera profesional, etc.; que de una u otra forma destacan al momento que un trabajador desarrolla sus funciones al interior de la entidad con el propósito de cumplir a cabalidad con los objetivos institucionales de la entidad a la cual pertenecen. Quintero (2019), menciona que la persona o servidor, cuando trabaja en equipo y responde en el cumplimiento de metas y resultados con total libertad incluso en las decisiones que toma, necesita ser entrenado y recibir el fortalecimiento de capacidades que logre involucrarse en la selección de sus responsabilidades, crear ambientes de trabajo cooperante, compartir información, establecer en grupo los objetivos a donde quieren llegar utilizando todas sus capacidades y destrezas, con un nivel de confianza elevada por parte del equipo de trabajo, al final obtendrá el reconocimiento por el éxito alcanzado y estímulo por su alto desempeño laboral.

Del análisis realizado sobre el desempeño laboral, los trabajadores de las distintas áreas donde se desarrollan los estudios, refieren que ante la existencia de un buen clima organizacional y/o laboral, es factor clave para que las personas cualquiera sea su profesión el rendimiento o desempeño laboral al interior de sus organizaciones será siempre productivo y si es lo contrario, será la otra cara de la medalla, adversidades que las entidades deben mejorar a favor de sus trabajadores y clientes potenciales (Cabezas, 2016; Pilligua \& Arteaga, 2019; Moral \& Ganzo, 2018; Atencio \& Tarqui, 2020).

Moreno y Wong (2018), señalan que en la mayoría de organizaciones estatales prevalece un clima organizacional distinto al privado, las condiciones laborales presentan deficiencias a pesar de los años, los estados emiten una serie de disposiciones para modernizar sus entidades cuando la realidad es distinta, encontraron locales en condiciones que no permiten ofrecer una óptima calidad en los servicios de la salud, educación. Otro aspecto que juega un papel importante en los servidores del sector público, según Campos, y Matzumura (2019) no existe el reconocimiento al esfuerzo desplegado por los profesionales, las entidades no promueven ni ofrecen su desarrollo y apoyo, el centralismo es un arma que mata a las organizaciones de provincias, los procesos de supervisión han desaparecido y si se realizan no son parciales ni objetivas, las infraestructuras en el sector salud es deficiente, que no permite desarrollar su labor con eficiencia, condiciones que el mismo estado tiene responsabilidad al no poner énfasis en este sector y otros.

Jara, Asmat, Alberca y Medina (2018), sostenían que el desempeño deficiente se imputaba a la falta de conocimientos entre los teóricos y lo práctico, haciendo una reflexión meditada, depende de diversos 
aspectos que se caracterizan según el tipo de población y/o usuario atendido, los trabajadores, el sistema y el entorno determina las diversas condiciones en las que se desempeña el servidor público. Realizar actividades laborales en inadecuadas condiciones afecta la eficiencia y la eficacia de los procesos administrativos, los factores que disminuyen la capacidad de respuesta en las actividades, especialmente la salud, provocan ausentismo en los centros laborales, mostrando una deficiente calidad en los procesos exigidos por la sociedad actual (Quintero, Álvarez \& Ibagon, 2019).

La evaluación del desempeño laboral, en muchas entidades del sector público, no se realiza a pesar que las normas lo estipulan; es un problema o desafío generado por el mismo empleador en la gestión del recurso humano. En lo que resta del año no se realizan evaluaciones de desempeño a los trabajadores, existiendo brechas para medir los objetivos e indicadores institucionales en lo que respecta al recurso humano, obviando algunos factores base como es la cultura organizacional, lo atractivo de la organización, el uso de tecnologías en las tareas, leyes y valores de la sociedad, trayendo consecuencias a largo plazo como son el bienestar individual, la eficacia y la eficiencia en la organización y el bienestar social, a través de los grupos de interés compuesto por los empleados, directivos, sindicato, gobierno, sociedad y la humanidad al interior de la organización.

En tal sentido, el objetivo de esta investigación fue evaluar las competencias de los servidores como factor clave de su rendimiento.

\section{MATERIALES Y MÉTODOS}

La metodología aplicada fue la revisión bibliográfica, los tipos de estudios realizados, predominó el descriptivo de corte transversal con un enfoque cuantitativo y diseño no experimental. Mediante la aplicación de encuestas, utilizando la escala tipo Likert con los niveles que más se adaptaron al estudio a desarrollar. La recopilación de algunas fuentes se realizó a través artículos cuya especialización estuvo acorde a la necesidad de las investigaciones. Se observó que los autores de las investigaciones tuvieran en cuenta las consideraciones éticas, al solicitar el consentimiento informado verbal a cada participante.

\section{RESULTADOS}

Del análisis realizado, se evidenció que, del total de personas encuestadas cuentan con insuficiente desempeño, en su mayoría expresó que no fueron capacitados por la entidad (valor=0,03). Se seleccionó las respuestas señaladas en la Tabla 1, que conlleve a un similar diagnóstico que se quiere difundir como resultado general. 
Tabla 1.Factores organizaciones y desempeño laboral

\begin{tabular}{|c|c|c|c|c|c|}
\hline \multirow{2}{*}{ Características organizacionales } & \multirow{2}{*}{$\begin{array}{c}\text { Valor } \\
\mathbf{p}\end{array}$} & \multicolumn{2}{|c|}{ Insuficiente } & \multicolumn{2}{|c|}{ Suficiente } \\
\hline & & $\mathbf{N}$ & $\%$ & $\mathbf{N}$ & $\%$ \\
\hline \multicolumn{6}{|l|}{ El ambiente laboral tiene todas las áreas } \\
\hline No & 0.36 & 35 & 68.6 & 118 & 75.2 \\
\hline $\mathrm{Si}$ & & 16 & 31.4 & 39 & 24.8 \\
\hline \multicolumn{6}{|l|}{ El ambiente es apropiado al tamaño } \\
\hline No & 0.22 & 40 & 78.4 & 109 & 69.4 \\
\hline $\mathrm{Si}$ & & 11 & 21.6 & 48 & 30.6 \\
\hline \multicolumn{6}{|l|}{ Condiciones de seguridad } \\
\hline Poco segura & 0.72 & 32 & 62.7 & 94 & 59.9 \\
\hline Segura & & 19 & 37.3 & 63 & 40.1 \\
\hline \multicolumn{6}{|l|}{ Tiene los equipos de protección personal } \\
\hline Nunca & 0.12 & 4 & 7.8 & 9 & 5.7 \\
\hline A Veces & & 16 & 31.4 & 75 & 47.8 \\
\hline Siempre & & 31 & 60.8 & 73 & 46.5 \\
\hline \multicolumn{6}{|l|}{ Capacitación por la institución } \\
\hline No & 0.03 & 42 & 82.4 & 104 & 66.2 \\
\hline $\mathrm{Si}$ & & 9 & 17.6 & 53 & 33.8 \\
\hline \multicolumn{6}{|l|}{ Reconocimiento al trabajador destacado } \\
\hline No & 0.48 & 34 & 66.7 & 96 & 61.1 \\
\hline $\mathrm{Si}$ & & 17 & 33.3 & 61 & 38.9 \\
\hline \multicolumn{6}{|l|}{ Hay respeto entre los trabajadores } \\
\hline Nunca & 0.73 & 11 & 21.6 & 28 & 17.8 \\
\hline A veces & & 22 & 43.1 & 77 & 49.0 \\
\hline Siempre & & 18 & 35.3 & 52 & 33.1 \\
\hline \multicolumn{6}{|l|}{ Trabajadores son responsable } \\
\hline Pocos & 0.26 & 12 & 23.5 & 40 & 25.5 \\
\hline La mayoría & & 31 & 60.8 & 77 & 49.0 \\
\hline Todos & & 8 & 15.7 & 40 & 25.5 \\
\hline
\end{tabular}

Fuente: Atencio \& Tarqui (2020)

Tabla 2, se comprobó que, del total de profesionales encuestados con insuficiente desempeño, el $54.9 \%$ señalaron que su sueldo es regular. En su mayoría respondieron que no hay disponibilidad de materiales de oficina, la sobrecarga de trabajo es evidente y constante, se sintieron desmotivados y su ubicación es en base a su espacialidad; en la mayoría de casos las diferencias fueron significativas (valor $\mathrm{p}<0,05)$.

Tabla 2.Factores motivadores y desempeño laboral

\begin{tabular}{cccccc}
\hline Motivadores del trabajo & Valor & \multicolumn{2}{c}{ Insuficiente } & \multicolumn{2}{c}{ Suficiente } \\
& $\mathbf{p}$ & $\mathbf{N}$ & $\mathbf{\%}$ & $\mathbf{N}$ & $\mathbf{\%}$ \\
\hline Percepción del sueldo & & & & & \\
Bajo & 0.03 & 16 & 31.4 & 83 & 52.9 \\
Regular & & 28 & 54.9 & 58 & 36.9 \\
Alto & & 7 & 13.7 & 16 & 10.2 \\
Disponibilidad de equipos & & & & & \\
No & 0.02 & 41 & 80.4 & 99 & 63.1 \\
Si & & 10 & 19.0 & 58 & 36.9 \\
\hline
\end{tabular}




\begin{tabular}{|c|c|c|c|c|c|}
\hline \multicolumn{6}{|c|}{ Disponibilidad de material de oficina } \\
\hline No & 0.18 & 13 & 25.5 & 56 & 35.7 \\
\hline $\mathrm{Si}$ & & 38 & 74.5 & 101 & 64.3 \\
\hline \multicolumn{6}{|c|}{ Trabajo sobrecargado } \\
\hline Siempre & 0.01 & 48 & 94.1 & 124 & 79.0 \\
\hline A Veces & & 3 & 5.9 & 33 & 21.0 \\
\hline \multicolumn{6}{|l|}{ Desmotivado } \\
\hline $\mathrm{Si}$ & 0.01 & 43 & 84.3 & 102 & 65.0 \\
\hline No & & 8 & 15.7 & 55 & 35.0 \\
\hline \multicolumn{6}{|c|}{ Oportunidad de crecimiento profesional } \\
\hline No & 0.40 & 19 & 37.3 & 50 & 31.8 \\
\hline $\mathrm{Si}$ & & 32 & 62.7 & 107 & 68.2 \\
\hline \multicolumn{6}{|c|}{ Ubicación de acuerdo a su especialidad } \\
\hline No & 0.03 & 12 & 23.5 & 18 & 11.5 \\
\hline $\mathrm{Si}$ & & 39 & 76.5 & 139 & 88.5 \\
\hline \multicolumn{6}{|c|}{$\begin{array}{l}\text { Recibe sugerencia de mejora de su jefe o } \\
\text { supervisor }\end{array}$} \\
\hline Nunca & 0.40 & 18 & 35.3 & 46 & 29.3 \\
\hline A veces & & 20 & 39.2 & 55 & 35.0 \\
\hline Siempre & & 13 & 25.5 & 56 & 35.7 \\
\hline
\end{tabular}

Fuente: Atencio \& Tarqui (2020)

También se escogió las contestaciones sobresalientes en la Tabla 3, para llegar a un semejante juicio que se intenta trasmitir como supuesto resumen general. Sobresalió que del total profesionales encuestados con insuficiente desempeño, la mayoría expresó que divisaron presión laboral por parte de la jefatura (valor $\mathrm{p}<0,02$ ) y no tuvieron facilidades en su horario de trabajo (valor $\mathrm{p}<0,02$ ).

Tabla 3. Influencia de la jefatura y desempeño laboral

\begin{tabular}{|c|c|c|c|c|c|}
\hline \multirow[t]{2}{*}{ Influencia de la jefatura } & \multirow{2}{*}{$\begin{array}{c}\text { Valor } \\
\mathbf{p}\end{array}$} & \multicolumn{2}{|c|}{ Insuficiente } & \multicolumn{2}{|c|}{ Suficiente } \\
\hline & & $\mathbf{N}$ & $\%$ & $\mathbf{N}$ & $\%$ \\
\hline \multicolumn{6}{|l|}{ Presión laboral de la jefatura } \\
\hline Siempre & 0.02 & 28 & 54.9 & 103 & 65.6 \\
\hline A veces & & 17 & 33.3 & 24 & 15.3 \\
\hline Nunca & & 6 & 11.8 & 30 & 19.1 \\
\hline \multicolumn{6}{|l|}{ Jefatura acepta sugerencias } \\
\hline Nunca & 0.76 & 8 & 15.7 & 23 & 14.6 \\
\hline A veces & & 23 & 45.1 & 80 & 51.0 \\
\hline Siempre & & 20 & 39.2 & 54 & 34.4 \\
\hline \multicolumn{6}{|c|}{ Jefatura tiene palabras de ánimo para Ud. } \\
\hline Nunca & 0.95 & 10 & 19.6 & 29 & 18.5 \\
\hline A veces & & 25 & 49.0 & 81 & 51.6 \\
\hline Siempre & & 16 & 31.4 & 47 & 29.9 \\
\hline \multicolumn{6}{|c|}{ Cuando está en error, recibe orientación de su jefe } \\
\hline No & 0.94 & 14 & 27.5 & 44 & 28.0 \\
\hline $\mathrm{Si}$ & & 37 & 72.5 & 113 & 72.0 \\
\hline \multicolumn{6}{|c|}{ Tiene facilidades en su horario de trabajo } \\
\hline No & 0.02 & 25 & 49.0 & 48 & 30.6 \\
\hline $\mathrm{Si}$ & & 26 & 51.0 & 109 & 69.4 \\
\hline Su jefe valora su esfuerzo & & & & & \\
\hline
\end{tabular}




\begin{tabular}{lccccc}
\hline No & 0.52 & 36 & 70.6 & 118 & 75.2 \\
$\mathrm{Si}$ & & 15 & 29.4 & 39 & 24.8 \\
Relación entre usted y jefatura & & & & & \\
Mala & 0.34 & 4 & 7.8 & 21 & 13.4 \\
Regular & & 23 & 45.1 & 78 & 49.7 \\
Bueno & & 24 & 47.1 & 58 & 36.9 \\
\hline
\end{tabular}

Fuente: Atencio \& Tarqui (2020)

\section{DISCUSIÓN}

Se evidencia que el clima organizacional es base fundamental para desarrollar un buen desempeño laboral, la falta de área complementarias en los ambientes, tamaño, seguridad y protección, son factores importantes que influyen en el desarrollo de las funciones del trabajador, evidenciando que el resultado es afín con lo vertido por Moral y Ganzo (2018), quienes publicaron su artículo influencia de la inteligencia emocional en la satisfacción laboral de los trabajadores españoles.

Recibir la capacitación oportuna, el reconocimiento y respeto de la entidad, lleva a que todo servidor cumpla con responsabilidad sus faenas diarias, funciones que son visibles y puestos a disposición a través de los documentos de gestión (Manual de funciones) desde el inicio de la relación laboral y que en forma permanente realiza sin contar otras que son trasladadas por el jefe del área de su competencia y se refleja con lo vertido por Cuesta y otros (2018) quiénes publicaron su investigación evaluación del desempeño, participación y gestión de recursos humanos en la empresa.

Otro factor fundamental es la motivación, la percepción de un sueldo acorde a la situación económica que cada país tiene es fundamental, la tenencia o capacidad adquisitiva de la persona permita cumplir sin dificultad alguna sus actividades cotidianas que en su conjunto forman un bloque equivalente con la disposición de los equipos y material de oficina. Idéntica situación se presenta con lo descrito por Moreno y Wong (2018) en su literatura relación de habilidades directivas y satisfacción laboral en la empresa Chicken King de Trujillo.

La sobre carga de tareas genera desmotivación, que se agrava con las pocas o nulas oportunidades para crecer profesionalmente, en muchos de los casos por atender favores políticos, la asignación de funciones no está supeditado a la formación o especialidad donde mejor se desempeña, acción que se vuelve insostenible cuando el jefe de área no comparte o ignora sugerencias de mejora que deber ser trasladados al trabajador, llevando a que el desempeño laboral de los trabajadores sea deficiente e incumpliendo los objetivos que la organización plantea como reto al final del periodo trimestral, semestral y anual (Pilligua y Arteaga, 2019). 
Si el gerente muestra su experiencia para conducir los destinos de la organización destacando la comunicación y trabajo en equipo, los resultados finales serán los más óptimos; sin embargo, si es lo contrario, como sucede según el resultado del análisis realizado al conjunto de estudios seleccionados, se observa que existe presión laboral por parte de la jefatura, no acepta sugerencias, carencia de comunicación, nula orientación, las facilidades en los horarios es abolida, no valoran los esfuerzos desplegados y la nula relación jefe-subordinado, complica el desempeño laboral de los trabajadores, el ambiente laboral es deficiente, muestra signos negativos de productividad, los estilos de liderazgo en esta parte del estudio juega un papel tan importante hacia el logro de la calidad de satisfacción del trabajador atención oportuna de los servicios al público usuario (Rodríguez y Lechuga, 2019).

\section{CONCLUSIONES}

Ante la existencia de problemas que impiden desarrollar una óptima labor por parte de los servidores públicos o privados, es importante que los gerentes o jefes de áreas de las distintas organizaciones plasmen esfuerzos considerables para alcanzar un destacado desempeño laboral. El conocimiento del trabajo, producción, responsabilidad, capacidad de liderazgo e identidad laboral son para los directivos de uso habitual en los organismos públicas o privadas, con relación a ello, correspondería extender su atención con las habilidades, destrezas y experiencia de los gerentes de estas entidades.

Es importante que todas las organizaciones retomen su papel con la implementación de programas de evaluación periódica a los servidores, de adiestramiento de capacidades que busquen fortalecer los conocimientos y prácticas del buen uso laboral, crear una política de incentivos económicos o reconocimientos que motive a los trabajadores, manejar competencias de relaciones interpersonales, de comunicación eficaz, entre otras, para lograr la satisfacción de los colaboradores y, por ende, un mejor desempeño laboral. 


\section{REFERENCIAS BIBLIOGRÁFICAS}

Atencio, D., \& Tarqui, C. (2020). The perfomance of the nursing professional in a Social Security hospital in Callao-Peru. Archivo de Medicina, 1-17.

Cabezas, E. (2016). Analysis of teachers burnout syndrome in the Faculty of Engineering of the National University of Chimborazo and its impact on jos perfomance. Producción y Gestión, 59-68.

Campos, P., Gutiérrez, H., \& Matzumura, J. (2019). Job rotatio and work perfomance of nurse practitioners at a specialized healthcare center. Cuidarte, 1-15.

Cuesta, A., Fleitas, S., García, V., Hernández, I., Anchundía, A., \& Mateus, L. (2018). Perfomance assessment, engagement and human resources management at enterprise. Ingeniería Industrial, 24-35.

Jara, A., Asmat, N., Alberca, N., \& Medina, J. (2018). Human talent management as a factor for improving public management and job perfomance. Revista Venezolana de Gerencia, 1-24.

Moral, M., \& Ganzo, S. (2018). Influence of emotional inteligence in job satisfaction in Spanish workers. Psicología desde el Caribe, 1-17.

Moreno, M., \& Wong, H. (2018). Relationship of managerial skills and job satisfaction in the company chicken king de Trujillo, 2018. Cuadernos Latinamericanos de Administración, 1-17.

Pilligua, C., \& Arteaga, F. (2019). The labor climate as a key factor in the productive performance of companies. case study: Hardepex Cía. Ltda. Radalyc, 1-25.

Quintero, L., Álvarez, C., \& Ibagon, D. (2019). Domestic violence in the labor perfomance of women in the textile-confection industry. Diversitas: Perspectivas en Psicología, 271-284.

Rodríguez, P., \& Lechuga, J. (2019). Work perfomance of teacers at ITSA University Institution. Escuela de Administración de Negocios, 79-101. 